\title{
Proceedings of the 128th Semon Club, Otolaryngology Department, Guy's and St Thomas' NHS Foundation Trust, London, UK, 19 November 2004
}

\author{
Chairman: Ms E Chevretton, Consultant Otolaryngologist, Guy's and St Thomas' NHS Trust, London \\ Pathologists: Professor L Michaels, University College London, and Dr I Clarke, University College London \\ and The Royal Free Hospital, London \\ Radiologist: Dr G Rottenburg, Consultant Radiologist, Guy's and St Thomas' NHS Trust, London \\ Secretary: Mr F Vaz, Specialist Registrar, Guy's and St Thomas' NHS Trust, London
}

\section{Paediatric session}

Chairman: Mr G Morrison

\section{Sialoblastoma (embryoma)}

C E B Giddings, D H Gadhvi, G A J Morrison

From Guy's and St Thomas' NHS Trust, London, UK

\section{Case report}

A sialoblastoma (embryoma) of salivary gland origin presented perinatally as a $5 \times 4 \mathrm{~cm}$ neck mass in the right digastric triangle. It did not cause airway compromise or feeding difficulty. Magnetic resonance imaging of the mass revealed it to be centred on the right submandibular gland. Fine needle aspiration cytology was suggestive of 'salivary gland anlage tumour'. Surgical excision through a submandibular approach was performed, without neurological deficit. Histopathological analysis revealed the mass to be a sialoblastoma. Excision margins were clear. Four months post-surgery, there was no evidence of recurrence.

There have been fewer than 20 cases of perinatal salivary gland tumour reported in the English literature. Sialoblastomas are the commonest of these tumours, and arise more commonly in the parotid than the submandibular gland. They appear locally aggressive but are considered benign. Histologically, sialoblastoma has a similar appearance to a primitive state of salivary gland development. There is arrest of salivary gland maturation, prior to acinar formation. Sialoblastomas show a significant degree of variability regarding their histology and clinical course.

The consensus for treatment is surgical excision alone, but there are very few previously described cases to guide the clinician.

\section{Discussion}

The forum discussed the diagnosis of truly congenital masses and the importance of differentiating radiologically between solid and cystic masses. The general consensus was that follow up should be for a few years.

\section{Aural growths and abdominal groans}

A V Patel, J Ahmed, J Kanagalingam

From the Chelsea and Westminster Hospital, London, UK

\section{Case report}

A two-year-old child presented with a chronically discharging right ear, resistant to antibiotics. Otoscopy revealed an aural polyp, and distraction testing suggested a conductive hearing loss of $35-40 \mathrm{~dB}$. The child also suffered from non-specific abdominal pain, constipation and peri-anal soreness. Sigmoidoscopy and large bowel biopies by paediatric gastroenterologists proved inconclusive.

An aural polypectomy yielded inflammatory tissue. A second aural polypectomy was undertaken for recurrence when an anterior attic defect was noted. Urgent computed tomography (CT) and magnetic resonance imaging scans showed a large area of abnormal soft tissue arising from the right temporal bone and the floor of the right middle cranial fossa, involving the dura and eroding the clivus.

The second histological specimen showed Langerhan's cell histiocytosis. At the time of writing, the child was receiving chemotherapy for multi-system Langerhan's cell histiocytosis at a national paediatric oncology centre. The current classification of the condition formerly known as 'histiocytosis X' was discussed.

\section{Discussion}

The differential diagnosis was discussed and the use of plain X-rays was suggested, as multiple lesions are very often seen on plain films. These are cheap, quick and simple, and much more accessible than CT scans.

\section{Rapid unilateral loss of sight in a child presenting with squint \\ C Blaine, S Lo, H Daya \\ From St George's Hospital, London, UK}

Case report

A four-year-old boy was referred routinely to the ophthalmology department following the diagnosis of a squint, which his mother had first noticed a few weeks earlier. There was a two-month delay in seeing an ophthalmologist, by which time the child had a decreased range of eye movements, mild proptosis and papilloedema. Three days later, the proptosis had increased and the patient had developed eye pain and headaches. An urgent referral was made to the paediatric oncology service, who noted severely impaired vision in the right eye. Magnetic resonance imaging scans showed a retro-orbital mass extending into the ethmoid cells. The patient received an empirical dose of vincristine and dexamethasone in an attempt to save his vision, on the presumption that the lesion was neoplastic. Endoscopic examination revealed a right nasal polypoidal lesion, which was biopsied under general anaesthetic. Histological analysis confirmed the diagnosis of non-Hodgkin's lymphoma, and 
chemotherapy was commenced. Unfortunately, there was permanent loss of vision in the right eye. At the time of writing, the patient was still under review.

\section{Discussion}

Radiologically, it was thought that the lymphoma was probably centred orbitally rather than sinonasally. Questions were raised about the pre-biopsy chemotherapeutic treatment, and also about the need for quicker and closer liaison with all specialities when treating lesions in and around the orbit. The possibility of early steroid treatment was raised; this may have benefited eyesight preservation.

\section{Otology and skull base session}

Chairman: Professor M Gleeson

\section{When a parotid lump is more than a parotid lump}

L Liew, J Chan, C Barbaccia

From the Medway Maritime Hospital, Gillingham, UK

\section{Case report}

A 76-year-old man presented with a three-week history of an enlarging, asymptomatic left parotid lump. There were no other symptoms, nor any past medical or family history. The lump was $2.5 \mathrm{~cm}$, mobile, smooth and firm and was located in the tail of the parotid. Fine needle aspiration cytology was non-diagnostic. Magnetic resonance imaging (MRI) was consistent with a pleomorphic adenoma, but also revealed a left vestibular schwannoma and multiple meningiomas. A left superficial parotidectomy was performed, with full preservation of the facial nerve.

Histological analysis confirmed a schwannoma. This, together with the MRI findings, confirmed a diagnosis of neurofibromatosis type two, according to the criteria of multiple meningiomas (i.e. more than two), unilateral vestibular schwannoma, and one of the following: neurofibroma, glioma, schwannoma or juvenile posterior subcapsular lens opacity.

\section{Discussion}

This case generated plenty of discussion, firstly about the site of the lesion, which potentially arose from the greater auricular nerve, autonomic nerves or a small branch of the facial nerve itself. The speed of growth and the possibility of a malignant peripheral nerve sheath tumour were also discussed. Further discussions regarding neurofibromatosis type two centred on the need to send histology to Gareth Evans in Manchester, and also on the need for a formal referral to a neurocutaneous centre with a specialist interest.

\section{An interesting case of Vogtlen Koyanagilen Harada syndrome}

A Sil, P Chatrath, D J Gatland

From the Southend General Hospital, Southend-on-Sea, UK

\section{Case report}

A 58-year-old woman presented with a two-month history of a bilateral sensorineural hearing loss, intermittent vertigo and tinnitus. Over 24 hours, she had developed an acute, painful loss of vision. Examination revealed normal otoscopy, and audiogram showed a 50-70 dB sensorineural hearing loss. The rest of the neuro-otological examination was normal.

Bilateral anterior uveitis was diagnosed. A magnetic resonance imaging scan of the brain and skull base was normal. Lumbar puncture showed mild lymphocytic pleocytosis. Autoantibody screening, erythrocyte sedimentation rate, syphilis serology and serum calcium levels were all normal.

The patient improved dramatically on intravenous dexamethasone, and was discharged on oral steroids. Her hearing improved by $25-30 \mathrm{~dB}$, and her visual acuity also improved.

However, three weeks after withdrawal of steroids, the patient developed recurrent, severe ocular symptoms, and presented with bilateral pan uveitis (anterior and posterior). The symptoms subsided with a further course of steroids, and the possibility of an autoimmune condition involving the ear and uveal tract was considered.

A presumptive diagnosis of Vogtlen Koyanagilen Harada syndrome was made, based on the diagnostic criteria set by the American Uveitis Society.

\section{Discussion}

Discussion centred around the rarity of bilateral hearing loss, and the fact that this was almost always related to an underlying systemic condition which must be investigated thoroughly.

\section{Chronic otorrhoea: Langerhan's cell histiocytosis of the petrous temporal bone}

R J D Hewitt, R Kuchai, M J Wareing

From St Bartholomew's Hospital and The Royal London Hospital, London, UK

\section{Case report}

A 32-year-old man with resistant otorrhoea and otalgia in the left ear was discovered on mastoid exploration to have systemic Langerhan's cell histiocytosis; this extended to the posterior canal wall and involved the mastoid antrum but spared the facial nerve.

A short initial post-operative period of improvement was followed by recurrence of symptoms. Aggressive treatment with two doses of radiosurgery was undertaken; however, serial magnetic resonance imaging (MRI) identified progressive disease within the petrous temporal bone. At the time of writing, the patient's main symptoms were progressive hearing loss and vertigo.

\section{Discussion}

The question of the benefit of intratympanic gentamicin perfusion was raised, but no consensus was reached. Certainly, the need for further imaging, in the form of MRI scanning to assess the lateral sinus, was suggested.

A further oncological opinion was thought appropriate. A request was made for a follow-up report to a subsequent Semon club meeting, in order to assess this patient's progress.

\section{An unusual case of intractable otitis externa}

R Ganesalingam, S Lo, T A Odutoye

From St George's Hospital, London, UK

\section{Case report}

A 58-year-old man presented with several months' history of left-sided, intractable otitis externa. The condition had failed to respond to topical antibiotics, and the patient denied any previous otological history. On examination, he had a small, ulcerated lesion in the floor of the left external auditory meatus. A local excision biopsy of the ulcer was performed, with a view to wider excision if malignancy was confirmed. The histological appearance was consistent with actinomycosis, with typical, filamentous organisms within a 'granule' seen on haematoxylin and 
eosin staining. A computed tomography scan showed an increased amount of soft tissue in the floor of the left external ear canal. The patient was commenced on oral co-amoxiclav $625 \mathrm{mg}$ thrice daily, and continued this for three months. The patient was reviewed at regular intervals for seven months. The ulcer healed completely without recurrence, and extensive surgery was avoided.

\section{Discussion}

Professor Michaels could not deny the fact that this was thought to be an actinomycotic infection, but expressed a wish to view more slides. The central message gleaned from this discussion was that external auditory canal lesions should be biopsied early and often.

\section{Differential diagnosis of parotid region masses}

T Kamani, R Thomas, N Violaris

From the Eastbourne District General Hospital, Eastbourne, UK

\section{Case report}

A 76-year-old woman presented with an irregular, hard, non-tender, immobile mass in her left pre-auricular region. Ultrasonography showed an irregular mass with calcification in the superficial parotid gland. A computed tomography scan revealed a calcified mass deep to the normal parotid gland and attached to the temporomandibular joint. A total parotidectomy and total excision of the tumour from the temporomandibular joint were performed. The post-operative course was uneventful

Histological analysis demonstrated chondrocalcinosis due to the presence of bifringent calcium pyrophosphate crystals and metaplastic cartilage. Chondrocalcinosis (also known as tophaceous pseudogout) may vary in presentation, from an incidental, asymptomatic finding to a severely destructive arthropathy, and may rarely simulate a neoplasm. Only 17 cases of chondrocalcinosis have been reported in the head and neck region. It is very uncommon for chondrocalcinosis to form a large mass, but the temporomandibular joint is characteristic. Although very rare, chondrocalcinosis should be considered in the differential diagnosis of parotid masses with such a presentation.

\section{Discussion}

Some forum participants expressed surprise that initially a true cut biopsy was undertaken for this lesion. Questions about the need for surgery were also raised, bearing in mind the patient's age.

\section{Head and neck session}

Chairman: Mr J-P Jeannon

Carcinoma of the right vocal fold, two years after therapeutic mitomycin use to prevent recurrence of an anterior web

I Friedrichs, M Yaneza, G A J Morrison

From Guy's Hospital, London, UK

Case report

A 67-year-old man presented in 1998 with an anterior laryngeal web, which had been treated by laser on five previous occasions. On the last occasion, in 2002, topical mitomycin was applied to the surgical site to prevent recurrence.

In 2004, the patient represented with another deterioration of his voice. On clinical examination, a polypoid lesion of the right vocal fold was found, in addition to an anterior laryngeal web. Biopsy revealed a squamous cell carcinoma of the right vocal fold, including the anterior laryngeal web. Following this biopsy, the cancer was successfully treated with laser excision and post-operative radiotherapy.

Recently, mitomycin, a well established chemotherapy drug, has been used topically in the treatment of laryngeal stenosis, and has increased the rate of successful outcome from 20 to 75 per cent. Chemotherapy drugs can cause secondary malignancies; however, when used for benign laryngeal disease, malignant change has not been reported.

\section{Discussion}

Professor Michaels confirmed the diagnosis of squamous cell carcinoma but expressed a desire to view the earlier sections from this case. There was much discussion about the potential of mitomycin to induce malignancy following topical application.

\section{An unusual neck mass with a future}

D E J Whitehead, D Gillett, J Topham From the Royal Sussex County Hospital, Brighton, UK

\section{Case report}

A 38-year-old man presented with a left-sided neck mass that had gradually increased in size over the course of a year. He complained of intermittent dysphonia, although he denied any odynophagia or dysphagia. He was a nonsmoker and drank alcohol occasionally. Palpation of the neck revealed a $3 \mathrm{~cm}$, firm, non-tender, fully mobile mass at the level of the left thyroid cartilage. A magnetic resonance imaging scan revealed a $4.5 \mathrm{~cm}$ mass adjacent to the submandibular gland, with further nodes lying posteriorly. An excisional biopsy was recommended to exclude lymphoma. At surgery, an encapsulated lesion behind the sternomastoid muscle was excised. Clinically, this had the appearance of a branchial cyst. Histological analysis revealed an enlarged lymph node with very abnormal architecture. An expert review was required to confirm the diagnosis of plasmacytoma.

\section{Discussion}

Professor Michaels pointed out that it was essential to view the histology slides in order to make a complete evaluation of the histological diagnosis, as a wealth of differentials could be included. He also pointed out that the diagnosis of a solitary neck plasmacytoma has clearly been defined as a separate, unrelated entity to myeloma.

\section{Airway obstruction due to laryngeal paraganglioma \\ E Kiverniti, C C Giddings, T Odutoye \\ From St George's Hospital, London, UK}

\section{Case report}

An adult female patient presented with airway obstruction secondary to a large, right-sided, supraglottic mass of the larynx. This was initially diagnosed as a haemangioma, and angiography with embolisation of the tumour produced a marked involution, along with resolution of the patient's symptoms. Recurrence of the mass, with its symptoms, precipitated the need for surgery via a lateral pharyngotomy approach. This was complicated by severe haemorrhage, and the patient underwent ligation of the right external carotid artery. Histological analysis confirmed a typical Zellballen pattern of the cells, and chromogranin staining suggested a paraganglioma. This is a tumour of neuroendocrine origin, similar to those originating from the carotid body. The excision was incomplete. A later angiogram revealed multiple bilateral feeding vessels, not amenable to embolisation, and so the patient 
underwent a second operation via a transhyoid approach and laryngeal fissure. The tumour was macroscopically completely excised, but histology revealed incomplete microscopic margins. The patient underwent a course of external beam radiotherapy, and at the time of writing was currently under close, long-term follow up.

\section{Discussion}

The possibility of endoscopic resection for local control were discussed. There was also some debate about the need for radiotherapy; the conclusion was that its role is actually unclear at the present time.

\section{An interesting case of asthma?}

A Sil, P Chatrath, G Watters

From the Southend General Hospital, Southend-on-Sea, UK

\section{Case report}

A 32-year-old man presented to the accident and emergency department with rapidly progressive dyspnoea. He was a smoker with no previous chest problems. Respiratory examination revealed extensive wheezing which partially responded to nebulised salbutamol. A working diagnosis of asthma was made, and he was admitted for further treatment involving systemic steroids, nebulisers and bronchodilators. He continued to deteriorate, with intermittent episodes of dramatic desaturation.

An ENT opinion was sought, and flexible nasendoscopy showed an apparently normal supraglottis and larynx. A chest X-ray demonstrated a sizeable pneumomediastinum. A computed tomography scan of the chest showed a $1.5 \mathrm{~cm}$ mass in the subglottis, extending into the trachea. An emergency tracheostomy under local anaesthetic was performed, and laryngoscopy revealed a firm to hard mass almost completely occluding the subglottic space. Histological analysis confirmed the presence of a poorly differentiated anaplastic small cell carcinoma, with extensive peritracheal lymphatic involvement. Following a period of counselling, the patient commenced a regime of chemotherapy followed by radiotherapy.

\section{Discussion}

The ensuing discussion highlighted the importance of securing an airway before further investigations, and this was emphatically agreed on by the forum. Also highlighted was the need for investigation of the 'unresponsive asthmatic'. Participants expressed some surprise that such a large subglottic mass was not visualised at nasendoscopy, as this lesion lay just within the subglottis.

\section{Recurrent hyalinising clear cell carcinoma}

J Rimmer, P A Harris, P M Clarke

From The Royal Marsden Hospital, London, UK

\section{Case report}

A 67-year-old man had presented initially with a right buccal mucosal lesion. A biopsy had been reported as myoepithelial carcinoma, which had been treated with radical radiotherapy. After eight years, a progressively enlarging swelling at the same site was noted. Biopsy of this lesion diagnosed hyalinising clear cell carcinoma of salivary gland origin. The patient was referred to our hospital.

Symptoms included right facial swelling, trismus and diplopia on right lateral gaze. A computed tomography scan confirmed an extensive recurrence involving the maxilla, zygomatic arch, infratemporal fossa, orbit and temporomandibular joint. A right maxillectomy, partial mandibulectomy and orbital exenteration were performed with a free rectus abdominus flap reconstruction.

Clear cell carcinoma of the salivary glands accounts for less than 1 per cent of all salivary tumours. Hyalinising clear cell carcinoma is an extremely rare subtype which requires immunohistochemical analysis for accurate diagnosis. Hyalinising clear cell carcinoma has a female preponderance and most commonly arises from the minor salivary glands. The tumour is low grade, locally invasive and rarely metastasises. Treatment is by complete local excision.

\section{Discussion}

It was clear in the discussion that post-radiotherapy cases required radical surgery in order to achieve any chance of cure. Some discussion centred on the rationale for orbital sacrifice, and this was deemed completely appropriate in view of the infiltration of the lateral rectus muscle.

\section{Tuberculous thyroid abscess with tracheal compression}

D H Gadhvi, C Skilbeck, R Simo

From the Lewisham Hospital, London, UK

\section{Case report}

A 64-year-old Asian woman developed a one-week history of fever and neck pain. She presented as an emergency case with a compromised airway which required intubation. Clinically, a large, smooth goitre was palpable, with retrosternal extension. Computed tomography showed a large, right-sided mass extending into the mediastinum, causing displacement and compression of the trachea. Surgical exploration revealed a large thyroid swelling which discharged $100 \mathrm{mls}$ of pus when the capsule was opened. Microbiological cultures grew Mycobacterium tuberculosis. The patient was started on anti-tuberculous therapy.

\section{Discussion}

Following this presentation, the interesting point was raised that this may possibly have been a secondary event due to vertebral tuberculosis, and that further imaging may well have been of benefit. The possibility of ultrasound-guided aspiration as a method of relieving some of the pressure on the airway was discussed; however, it was agreed that, in the case of such a large abscess, a formal, open drainage procedure was appropriate.

\section{Rhinology session}

Chairman: Mr D Roberts

\section{Image-guided endoscopic extraction of a posterior nasopharyngeal wall airgun pellet \\ T Maani, D Hajioff, H Saleh \\ From the Charing Cross Hospital, London, UK}

\section{Case report}

A 28-year-old man attended his local accident and emergency department after shooting himself in the mouth with an airgun.

Initial assessment revealed a right-sided oral commissure laceration and a hole in the right side of the soft palate. A lateral cervical spine X-ray followed by a computed tomography scan showed a pellet lodged in the posterior nasopharyngeal wall, just inferior to the sphenoid sinus. Initial endoscopic exploration failed to retrieve the pellet, as severe oedema of the post-nasal space made intra-operative localisation extremely difficult.

The patient was transferred to our hospital's otolaryngology department, where endoscopic extraction of the 
pellet was successfully performed using the BrainLAB Vectorvision ${ }^{\circledR}$ image-guidance system.

Image-guided endoscopic surgery appears to reduce complications in anatomically challenging sites. Our case illustrates a novel use for this technology, employed when conventional methods had failed.

\section{Discussion}

This case generated much discussion about the increasing usage of image guidance systems. They were deemed an essential part of the management of complicated sinonasal surgical cases, but were certainly not thought to be mandatory for all units.

\section{Intracranial polyps in patients with Samter's triad}

A Majithia, T Tatla, H Saleh, P Clarke

From the Charing Cross Hospital, London, UK

\section{Case report}

We report three patients with Samter's triad who had dehiscence of the skull base and posterior wall of the frontal sinus secondary to polyps. In two patients, the polyps extended intracranially into the extradural space. In the third patient, the polyps had gone through dura and were in direct contact with the frontal lobes. One patient unfortunately died in a road traffic accident while awaiting surgery. The other two underwent resection of the polyps via a combined frontal craniotomy and endoscopic approach. Histological analysis confirmed benign, eosinophilic polyps with no evidence of allergic fungal sinusitis. Neither of the patients developed a cerebrospinal fluid leak or meningitis, and no revision procedures were required (after follow up of six months and seven years). At the time of writing, both patients were well symptomatically.

This series shows that, in Samter's triad cases, nasal polyps can be very aggressive, with intracranial spread, but that this can be managed effectively with resection via a combined frontal craniotomy and endoscopic approach.

\section{Discussion}

The possibility of fungal rhinosinusitis was raised due to the appearances on the computed tomography scans. Unfortunately, the histology slides could not be reviewed and so no further comment could be made. It was also noted that there was a possibility of an endoscopic approach followed by aggressive medical treatment in this case. Significant questions were raised about the benefits of immunotherapy for aspirin-sensitive patients. The use of antileukotrienes in the management of such patients, after operative intervention, was deemed reasonable.

\section{'Don't turn a blind eye'}

I Friedrichs, D Roberts

From Guy's Hospital, London, UK

\section{Case report}

A 31-year-old woman had presented initially with reduced vision in her right eye, due to an enterogeneous cyst compressing her right optic nerve. She had been treated in another hospital (in 2002) with excision of the cyst via an open approach, which had initially improved her vision.

Enterogeneous cysts are extremely rare, intraorbital, congenital cysts containing a single layer of mucin-secreting epithelium resembling gastrointestinal epithelium.

In 2004, the patient represented with a recurrence of the cyst. She was treated with endoscopic orbital decompression and an attempt to remove the cyst under computer guidance. At the time of the operation, the cyst could not be identified, but this procedure improved her vision once more.

\section{Discussion}

It was concluded that this was a difficult case requiring a conservative approach, in view of the benign histology. A joint approach, with both an ophthalmologist and an ENT surgeon, was deemed suitable. The need to consider a neurosurgical approach was also highlighted, in view of the very difficult access to this particular cyst.

\section{Atypical presentation of Wegener's granulomatosis, with hard palate and nasal septum destruction}

E Ofo, S H Liyanage, J Marais

From the Northwick Park Hospital, Harrow, UK

Case report

A 23-year-old Asian woman presented with a six-month history of bilateral nasal obstruction and intermittent epistaxis. A small septal perforation with regular margins was noted on examination. Shortly after the initial consultation, she complained of intermittent central facial pain, and a foul odour from the mouth, with nasal regurgitation.

Examination revealed a $3 \mathrm{~cm}$ fistula in the hard palate, extending into the nasal cavity. The septal perforation was large, resulting in a saddle nose deformity. Blood tests showed normal renal function, and the erythrocyte sedimentation rate was not elevated. Tests for anti-neutrophil cytoplasmic antibody and syphilis serology were negative. The chest radiograph was normal, and computed tomography scans of the paranasal sinuses illustrated the absence of the nasal septum as well as mucosal thickening in both maxillary antra. Histological samples of the nasal septum showed necrosis and vasculitis suggestive of Wegener's granulomatosis

A diagnosis of 'limited' Wegener's granulomatosis was made, and the patient was referred to the rheumatologists, who commenced immunosuppressive therapy. Despite immunosuppressants, the palatal lesion continued to increase in size. The patient became positive for perinuclear anti-neutrophil cytoplasmic antibody five months after commencing treatment, but the erythrocyte sedimentation rate remained normal. The hard palate sequestrum was subsequently excised, and a palatal obturator used to cover the resultant oro-nasal fistula.

At the time of writing, the patient was no longer receiving immunosuppressants, and her renal function, erythrocyte sedimentation rate and C-reactive protein level continued to remain normal, with a negative antineutrophil cytoplasmic antibody test.

\section{Discussion}

Professor Michaels found the histology in this case unconvincing, and even raised the possibility of a midline lethal granulomatous process. It is not unreasonable to reconstruct the nose with a rhinoplasty, if inflammatory markers have been stable for two years. Autologous material seemed to be the most appropriate choice.

\section{Spontaneous cerebrospinal fluid rhinorrhoea: challenges in the diagnosis of dural defect}

C Neumann, K Desai, R M Tranter

From the Royal Sussex County Hospital, Brighton, UK

\section{Case report}

A 51-year-old man presented with a five-month history of watery rhinorrhoea, predominantly from the right nostril. The past medical history was unremarkable and the examination of the nose, ears and throat normal.

In view of the unilateral nature of the patient's symptoms, a spontaneous cerebrospinal fluid (CSF) fistula was 
suspected. A computed tomography (CT) scan of the paranasal sinuses and a magnetic resonance imaging scan revealed a thin cribriform plate but no breach. Nasal fluid tested positive for asialo-transferrin. A CT cisternogram demonstrated no communication with the nasal cavity or paranasal sinuses. An intrathecal injection of fluoresceine followed by endoscopic examination of the nasal cavities failed to identify the site of leak.

\section{Discussion}

This very interesting case provoked much discussion, but it was strongly suggested that an endoscopic exploration of the skull base would be appropriate. However, it was certainly noted that, in cases of CSF rhinorrhoea, a complete and thorough evaluation of the ears should be undertaken.

\section{A congenital nasal mass presenting in adult age with visual disturbance}

S H Liyanage, A Majithia, D D A Lawson, J Grieve

From the Northwick Park Hospital, Harrow, and The National Hospital for Neurology \& Neurosurgery, London, UK

\section{Case report}

A 41-year-old woman presented to the physicians with a one-week history of visual disturbance. Examination revealed bilateral proptosis with associated impairment in visual acuity (6/60 bilaterally) and colour vision. Computerised tomography scanning of her orbits showed severe, bilateral optic nerve swelling and a mass in the left nasal cavity extending through the cribriform plate. Following a course of intravenous steroids, the patient's visual symptoms resolved. Her care was transferred to the ENT department, where further questioning revealed a preceding three-month history of mild, left-sided nasal obstruction and rhinorrhoea. Magnetic resonance imaging revealed absent flow in the anterior part of the superior sagittal sinus. The mass emanated from the lateral nasal wall and was noted to be pale, firm and nonpulsatile on rigid nasendoscopy. Its size did not vary during the Valsalva manoeuvre, and the Furstenberg test was negative. A histological sample identified the lesion as heterotopic neuroglial tissue. The patient was transferred to the regional neurosurgical unit, where her symptoms quickly settled and a conservative approach was adopted.

\section{Discussion}

Some surprise was expressed that surgical excision had not been offered to the patient. It was suggested that a formal referral be made to a craniofacial unit, such as that at the Chelsea and Westminster Hospital, London. 